Vierteljahrsschrift für Geschichte der Medizin und der Naturwissenschaften

Revue trimestrielle d'histoire de la médecine et des sciences naturelles

Redaktion/Rédaction:

Carl Haffter, Basel

\section{GESNERUS}

Jahrgang/Vol.40 1983

Heft/Fasc. 3/4

Verlag/Editions Sauerländer, Aarau

\title{
Herkunft und Schicksal des Humor aqueus
}

Physiologische Untersuchungen im Vorfeld der Ätiologie des Glaukoms während des ersten Viertels des 20. Jahrhunderts*

Von Gerhard Rudolph

\begin{abstract}
Communis, puto, ut aliorum humorum, ita hujus aquae ex arteriis est scaturigo. Neque rarum exemplum est, aut impulsum in oculi vasa terebinthinae oleum in aqueum humorem exire, aut argentum vivum (Vieussens) ad cutem illitum, aut per vasa impulsum (Hovius, Zinn), aliumve liquorem in eum humorem se admiscuisse. - Quae arteriae eum humorem generent, non penitus potest definiri.
\end{abstract}

A.v.Haller, Elementa Physiologiae T. V, sensus externi, 1769

Lehrbuchmäßig vereinfachend hat Haller (in der Übertragung von Sömmering 1788) den Sachverhalt so dargestellt: «... in dem kleinen dreieckigten krummlinigten Raum hinter der Blendung und Linse, und in dem größern Segment der holen Kugel zwischen der Blendung und Hornhaut, schwimmt die wäßrige Feuchtigkeit, die höchst flüssig und durchsichtig, und nach einem Verlust ersetzbar ist. Sie scheint aus den Arterien der Blendung und der Ciliarfortsätze ausgehaucht, und in ähnliche Venen wieder eingesaugt zu werden, auch dünstet vielleicht ein Theil, der in der Hornhaut eingesaugt wird, durch sie aus. Mit eben der Feuchtigkeit wird die hintre Seite der Blendung und die Kapsel der Linse benetzt. Kammern des Auges nannte man zu Anfang dieses Jahrhunderts die Räume, die von dieser Feuchtigkeit ausgefüllt werden ...»

\footnotetext{
*Herrn Prof. Huldrych M. Koelbing zum 60. Geburtstag gewidmet.
} 
Die Physiologie des 18. Jh. hat der Formerhaltung und der Ernährung des Auges einen verhältnismäßig großen Raum gewidmet, der ihrer Morphologiebezogenheit entsprach, auch wenn sie spekulative Überbewertungen der «liqueurs» («tout ce qu'il y a de sensible dans la machine animale»), wie in der Sinnesphysiologie (1767) von Le Cat (1700-1768), mit hallerischer Nüchternheit zurückwies. Die Physiologie diente aber zugleich der Medizin, die Sehstörungen, wie sie durch Veränderungen der lichtdurchlässigen Medien des Auges verursacht werden, ex officio nachzugehen hatte. So findet sich eine bemerkenswerte Häufung medizinisch-naturwissenschaftlicher Untersuchungen zur Frage der Unterscheidung von Glaukom und Katarakt zu Beginn des 18. Jh. mit dem Bemühen, über die ätiologischen Vorstellungen von Galen hinauszukommen. Dahin gehören die Ansätze von Jean Mery (1645-1722), von Michel Brisseau (gest. 1743), die ihren Niederschlag in «Histoire» und «Mémoires de l'Académie royale des Sciences» gefunden haben. Dahin gehören aber auch Arbeiten von Jakob Hovius (1716), von Pierre-Simon Rouhault (gest. 1740), vielleicht auch spätere Beobachtungen von Jacques Daviel (1693-1762) (Vetter 1963), sowie rangmäßig sicher nicht zuletzt der Nachweis gleicher Brechungsindices von Humor aqueus und Glaskörper im Rinderauge (1707) durch PhilippeGabriel De la Hire (fils) (1677-1719). Diese Untersuchungen, sowie Arbeiten «sur la nature des cataractes» waren durch das Interesse des Vaters an den «différents accidents de la vue», hervorgehoben in den Eloges von Fontenelle, vorgezeichnet.

Mit der Ausbreitung des physikalischen Denkens in der Physiologie des 19. Jh. (Rudolph 1983), welche vitalistische Strebungen, aber auch primär klinische Fragestellungen als Ausgangspositionen für ihre experimentellen Programme verwirft, kommt es zu einer Interessenverschiebung auf dem Gebiet des Gesichtssinnes in Richtung einer stärkeren Betonung der geometrischen Optik (Abbildungsgesetze, Akkommodation) und der Erregungsphysiologie (visuelle Informationsverarbeitung). Die Vorlesungen über «die neueren Fortschritte in der Theorie des Sehens» (1868) von Hermann Helmholtz (1821-1894) kennzeichnen solche Einstellung. Sie gilt in gleicher Weise für Jean-Pierre Morat (1846-1920) in seinem Traité de Physiologie (Fonctions de relation 1918).

In Lehrbüchern der Physiologie, die über einen Teil des 20. Jh. führend blieben, wie die von Rudolf Höber (1919, 8. Aufl. 1939) oder Hermann Rein (2.Aufl. 1938) fehlt jede Erörterung der Frage des intraokularen Flüssigkeitswechsels. Erst in der Bearbeitung von Max Schneider (16. Aufl. des 
Rein 1971) oder dem Beitrag von O.-J. Grüsser (20. Aufl. 1980) taucht das Stichwort des Augeninnendrucks mit einer kurzen, die Diskussionen der Physiologie nicht berücksichtigenden Erklärung wieder auf. Eine Ausnahme macht ein Hauptvertreter der "physikalischen Richtung» Ludimar Hermann (1838-1914) der in seinem über das reine Physikum-Interesse weit hinausgehenden Lehrbuch (hier 13. Aufl. 1905, 14. Aufl. 1910) den «Blutumlauf im Augapfel», "Chemie und Erneuerung der Augenmedien», den «intraokulären Druck» ausführlich behandelt.

Mit der physiologischen Interessenverschiebung in Richtung der Aufklärung des normalen Sehvorgangs mittels Gewinnung präziser Daten aus der physiologischen Optik (Helmholtz) bis zu der Aufdeckung der molekularen Grundlagen der Photorezeption, wechselt der Forschungsgegenstand «Augenflüssigkeiten» vollends über in den klinischen und experimentellen Bereich der Ophthalmologie. Denn die Fragen nach dem Wasser- und Stoffaustausch, nach Entstehung und Aufrechterhaltung des intraokularen Drucks sind von grundlegendem klinischen Interesse für Genese und mögliche Therapie des akuten Glaukoms. 1855 vermutet Albrecht von Graefe (1828-1870) die intraokulare Druckzunahme als Entzündungsfolge einer «Chorioiditis sensa» (Schmidt-Rimpler), 1857 behandelt er das Glaukom durch Iridektomie, um damit eine Produktionsstätte des Kammerwassers auszuschalten. William Arthur Brailey (1845-1915) sieht aufgrund anatomischer Untersuchungen die Ursache des Glaukoms mechanisch in einer Arterienerweiterung im Gebiet des Corpus ciliare.

Von weiter reichender Bedeutung werden die Arbeiten des GraefeSchülers Theodor Leber (1840-1917). Er hat, gestützt auf ältere Beobachtungen über die Möglichkeit, Störungen des intraokularen Drucks durch Flüssigkeitszufuhr oder -abfuhr auszugleichen, einen kontinuierlichen Wechsel der Augenflüssigkeiten angenommen und, in experimentellen Arbeiten, u. a. mit dem Straßburger Anatomen Gustav Albert Schwalbe (1844-1916), die Grundlagen geschaffen, die zur Formulierung der bekannten Leberschen Filtrationstheorie führten. Sie besagt, daß das Kammerwasser im Corpus ciliare andauernd gebildet wird und im Kammerwinkel über die Fontana'schen Räume und die Membranen des Sinus venosus Schlemmii und durch die Vorderfläche der Iris abfließt. Dieser ständige Flüssigkeitsstrom würde beim Menschen $0,3 \mathrm{ml} / \mathrm{h}$ betragen; in 48 Minuten wäre der Kammerinhalt vollständig erneuert. Lebers Befunde werden u. a. durch Versuche der Ophthalmologen Erich Seidel (geb. 1882) und Georges Leplat (geb. 1890) gestützt. Indessen hat das Regenerat andere chemische Eigen- 
schaften als der normale Humor aqueus. Carl Hamburger (1870-1944) hat schließlich nachgewiesen, daß die Neubildung nicht in der von Leber errechneten Größenordnung stattfinden kann und hat, einer persönlichen Anregung von Paul Ehrlich folgend (Fluoreszein-Injektionen), die Vorderfläche der Iris als normale Produktionsstätte des Humor bestimmt, während die Ciliarfortsätze nur bei größeren Kammerwasserverlusten einspringen sollten.

An dieser Stelle schaltet sich die Königsberger physiologische Schule mit ihren Bedenken gegen den kontinuierlichen Flüssigkeitsstrom im Auge ein. «Solange die mechanischen Bedingungen für die Bildung und den Abfluß des Humor aqueus nicht vollkommen klar sind, muß man vermuten, daß andere Kräfte als die Druckkraft den Wechsel des Humor bewirken» schreibt 1905 Otto Weiss (1871-1943), ein Schüler von Ludimar Hermann. Dieser war 1884 nach 16jähriger Tätigkeit in Zürich nach Königsberg übersiedelt, an ein Institut, das durch die Entdeckungen von Helmholtz ausgezeichnet war. Weiss nimmt sich, nach sorgfältiger theoretischer Vorbereitung in Form seines Handbuch-Artikels (1905), «mit dem ganzen Rüstzeug des geschickten Experimentators solchen etwas stiefmütterlich behandelten physiologischen Kleinfragen» an, wie sein Schüler Hans Lullies (1898-1982) später (1944) formulierte. Ich habe vergeblich bei Hermann einen vorbereitenden Fingerzeig in dessen auch das Auge (Farbensinn, Stereoskopie, Projektion der Netzhautmeridiane, Horopter) behandelnden Arbeiten gesucht, wenngleich eine persönliche Anregung bei seinem Interesse für das Thema plausibel erscheint. Die zunächst rein akademische Auseinandersetzung mit dem Problem bildete für Weiss Beweggrund genug für eine anschließende experimentelle Kritik, die fast zwei Jahrzehnte (1906-1924) erfüllte. Ihre Ergebnisse sind 1925 in einem Übersichtsartikel von ihm zusammengefaßt. Sie werden von Emil Abderhalden (1877-1950) im 3. Teil seines Lehrbuchs der Physiologie (1926) bereits ausführlich vorgestellt.

Nach Weiss reichen die Leberschen Versuche nicht aus, um den Schluß auf eine dauernde Neubildung und Abführung von Humor aqueus zu rechtfertigen, denn dies müßte am lebenden Auge einen ständigen Druckgradienten von der Bildungsstätte zum Schlemmschen Kanal voraussetzen. «... bei einem Injektionsdruck von der Höhe des normalen Augendrucks läßt sich weder Flüssigkeit in das Auge eintreiben, noch solche aus ihm herausleiten» (Weiss 1925). Es spricht also nichts für die Existenz eines dauernden $\mathrm{Zu}$ - oder Abflusses bei ungestörtem Gleichgewicht; vielmehr treten Kammerwasserbildung und Abfluß nur dann in Funktion, wenn 
Druckschwankungen auszugleichen sind. Die normale Aufgabe des Kammerwassers wäre rein mechanisch die eines hydrostatischen Puffers. Der bei einem Dauerstrom anzunehmende Stofftransport ist in Wirklichkeit so gering, daß diese statische Funktion ganz in den Vordergrund rückt (Abderhalden). Eine Druckdifferenz zwischen Kammerwinkel und hinterer Augenkammer wird auch ophthalmologischerseits von Carl Hamburger ausgeschlossen.

Schließlich hat Hans Lullies, der mir gegenüber einmal äußerte, daß es ihn wegen der mikrochirurgischen Aufgabenstellungen verlockt hätte, Augenarzt zu werden, in subtilen manometrischen Messungen mit einer verfeinerten Leberschen Methodik zeigen können, daß der Druck in den Abflüssen des Schlemmschen Kanals, welche intraskleral liegen, höher ist als der Augendruck. Mit dieser aus anatomischen Gründen notwendigen Ergänzung der Weissschen Kaninchenversuche am Hund (nur hier sind Anastomosen zwischen Kanal und Wirbelvenen) ist die Annahme eines Abflusses unter Wirkung von Druckkräften unmöglich geworden. Wie wäre aber die Erneuerung der Augenflüssigkeit, die doch sinnvollerweise postuliert werden müßte, zu erklären?

Hier hat der seit dem Ausgang des 19.Jh. erfolgte Brückenschlag zwischen Chemie und Physik neue Wege gezeigt, um die Gesetze zu erkennen, die das Geschehen des Lösungsbereichs beherrschen. Seit Beginn des Jahrhunderts orientierte sich die Physiologie an Rudolf Höber (1873-1953) «Physikalische Chemie der Zelle und Gewebe» (1902). 1911 stellt Leon Asher (1865-1943), der die Ionenzusammensetzung des Kammerwassers mitbestimmt hat, die auf die Physiologie anwendbaren physikalisch-chemischen Methoden in ihrer Breite vor und Heinrich Schade (1876-1935) setzt 1921 nur gleichsam den klinischen Schlußstein dieser auch für die Medizin folgenreichen Entwicklung.

So ist nach Weiss (1925) der unterschiedliche Elektrolyt- und Eiweißgehalt von Blutplasma und Kammerwasser mit einer Kammerwasserbildung durch Filtration nicht vereinbar. Es könnte hier aber der Schlüssel für das Verständnis der Beziehungen beider Flüssigkeiten zueinander liegen, in dem die nachgewiesenen Konzentrationsunterschiede (höhere Chlorid-Konzentration im Kammerwasser) im Sinne eines Donnan-Gleichgewichts zu deuten sind.

Lullies hat gezeigt, daß die Potentialdifferenz am Ciliarkörper nicht der Größe eines Sekretionspotentials entspricht, dagegen sehr wohl als Donnanpotential aufgefaßt werden kann. Hier wären also die «molekularen Kräfte» 
zu suchen unter deren Wirkung der Ausgleich zwischen Blut und Kammerwasser vor sich geht. W. Bladergroen hat diese Fragen des Donnan-Gleichgewichts zwischen Blut und Kammerwasser in seiner bewußt elementar gehaltenen «Physikalischen Chemie in Medizin und Biologie» unter Einbeziehung neuer Daten weiter erörtert (1949). Diese Erörterung wird in neueren ophtalmologischen Darstellungen der Kammerwasserzirkulation (Rohen 1969, Leydhecker 1980), vielleicht auf Grund erweiterter morphologischer Einsichten, nicht fortgesetzt.

Weiss schließt den Ergebnisbericht gemeinsamer Forschungen mit H. Lullies (1925) in wissenschaftlicher Bescheidenheit: «Wie die Filtrationstheorie und die Experimente, mit denen sie gestützt worden ist, Kinder ihrer Zeit waren, so sind es nicht minder die neueren Vorstellungen über das Wesen des intraokularen Flüssigkeitswechsels. Die biologischen Wissenschaften müssen nun einmal in den Fußspuren der Physik und Chemie wandeln und von ihnen Anregungen und Hilfsmittel entnehmen. So dürfen wir hoffen, daß mit dem Fortschreiten dieser Wissenschaften auch die eben erörterten Probleme immer weiterer Klarheit entgegengeführt werden.»

Die geschichtliche Betrachtung dieses umschriebenen Fragenkomplexes lehrt, daß der Erkenntnisgewinn in den Erfahrungswissenschaften nicht das Ergebnis spektakulärer Paradigmenwechsel ist, sondern eher ein Weg kleiner, korrigierender Schritte. Der erreichbare Horizont wird letztlich von den technischen Fähigkeiten des einzelnen Forschers bestimmt, mögen die intellektuellen auch unbegrenzt sein. So gelingt es, vielfach nur Teilaspekte zu erhellen, während andere Konturen wieder in das Dunkel sinken, z. T. durch einseitige Prägungen der wissenschaftlichen Lehre. In diesem Zusammenhang behält ein Satz von Huldrych Koelbing (1959) seine Gültigkeit: «Trotz imposanten Detailkenntnissen, zu deren Mehrung in jüngster Zeit die Berner Schule von Goldmann sehr viel beigetragen hat, sind wir bis jetzt weit entfernt von einer umfassenden und befriedigenden pathogenetischen Theorie des primären Glaukoms.»

\section{Bibliographie}

(allgemeine physiol. Lehrbuchhinweise im Text)

Asher, L.: Die Anwendung der physikalisch-chemischen Methoden in der Physiologie, in R. Tigerstedt (Hrsg.), Handbuch der physiologischen Methodik I, 2, 113, Leipzig 1911 Bladergroen, W.: Physikalische Chemie in Medizin und Biologie, 2. Aufl., Basel 1949 
Brisseau, M.: Traité de la cataracte et du glaucome, Paris 1709

Brailey, W. A.: s. Schmidt-Rimpler

Fontenelle, B. de: Eloge de M. de la Hire in Euvres T.V, 327, Amsterdam 1764

Haller, A.v.: Elementa Physiologiae Corporis Humani T.V. Sensus externi interni, Visus 306 s., Lausanne 1769

- Grundriß der Physiologie für Vorlesungen nach der 4. lat. Ausgabe besorgt von P.F. Mekkel, Berlin 1788

Hamburger, C.: Über die Ernährung des Auges, Leipzig 1914 Experimentelle Glaukomtherapie, Med. Klinik 19 (1923) 1224

Helmholtz, H.: Die neueren Fortschritte in der Theorie des Sehens, in Populäre wissenschaftliche Vorträge, 2. Heft, Braunschweig 1876

Höber, R.: Physikalische Chemie der Zelle und Gewebe, 6. Aufl. Leipzig 1926

Hovius, J.: De circulari humorum ocularium motu, Diss. Leiden 1716

La Hire, Ph. de: Remarques et réflexions sur la nature des cataractes qui se forment dans l'œil, Hist. Acad. Roy. Sci. 1706, 1707 p. 28 (1747)

Koelbing, H.M.: Die Begründung der Zellularpathologie durch Rudolf Virchow und die Augenheilkunde, Gesnerus 16 (1959) 124

Leber, Th.: Die Zirkulations- und Ernährungsverhältnisse des Auges in Graefe-Saemisch Handbuch der Augenheilkunde, 3 und 4, 1903

Le Cat, N.: Traité des sensations et des passions en général et des sens en particulier, Paris 1767 Leplat, G.: s. O. Weiss 1925

Leydhecker, W.: Glaukom in Axenfeld-Pau, Lehrbuch und Atlas der Augenheilkunde, S. 440 f., Stuttgart-New York 1980

Lullies, H.: Zur Zirkulation in den Venen des Auges, Diss. Königsberg 1922

- Der Druck in den Venen des Skleralrandes, Pflügers Arch. 199 (1923) 471

- u. L.Gulkowitsch: Beiträge zur Lehre vom Flüssigkeitswechsel des Auges, Schriften d. Königsberger gelehrten Ges. Naturwiss. Kl.1, H. 2 (1924) 105

- Otto Weiss (1871-1943) Erg. Physiol. (begründet von L. Asher u. K. Spiro) 45 (1944) 464

Méry, J.: Question de chirurgie, savoir: Si le glaucoma \& la cataracte sont deux différentes, ou une seule et même maladie, Hist. Acad. Roy. Sci. 1707 (1747) 654

Rohen, J. W.: Kammerwasserzirkulation (Morphologie), in K. Velhagen (Hrsg.), Der Augenarzt Bd.1, 106, Stuttgart 1969

Rouhault, P.-S.: s. J. Méry

Rudolph, G.: Ein Leben für die Physiologie - Prof. Dr. med. Hans Lullies zum 80. Geburtstag, Schleswig-Holsteinisches Ärztebl. 32 (1979) 12

- Das Mechanismusproblem in der Physiologie des 19. Jahrhunderts, Ber. Wiss. Gesch. 6 (1983), im Druck

Schade, H.: Die physikalische Chemie in der inneren Medizin, Dresden u. Leipzig 1921

Schmidt-Rimpler, H.: Glaucom in Eulenburg, Realencyclopädie d. ges. Heilkunde 8, 442

Seidel, E.: Methoden zur Untersuchung des intraokularen Flüssigkeitswechsels, in Abderhalden (Hrsg.), Handb. d. biol. Arbeitsmeth. Abt. 5, T.6, Berlin und Wien 1927

Vetter, Th.: Rencontres avec Jacques Daviel, Paris 1963

Weiss, O.: Die Ernährung und die Zirkulation des Auges in W.Nagel (Hrsg.) Handbuch d. Physiologie d. Menschen, Bd. 3, 438, Braunschweig 1905

- Die Lehre von der intraokularen Flüssigkeitsströmung ist nicht begründet, Pflügers Arch. 115 (1906) 174 
- Der intraokulare Flüssigkeitswechsel, Z. Augenheilk. 25 (1911) 1

- Der Druck in den Wirbelvenen des Auges, Z. Augenheilk. 43 (1921) 141

- Der Flüssigkeitswechsel des Auges, Pflügers Arch. 199 (1923) 462

- Über den Druck in den Venen des Bulbus, Pflügers Arch. 202 (1923) 642

- u. H.Lullies: Über den intraokularen Flüssigkeitswechsel des Auges, Pflügers Arch. 204 (1924) 763

- Herkunft und Schicksal der Augenflüssigkeiten, Dt. med. Wschr. 1925 I, 21 u. 63

\section{Summary}

Starting with a short review of research work undertaken in the XVIIIth and XIXth centuries to elucidate the origin and the circulation of Humor aqueus in the eye and the pathogenesis of glaucoma, the author describes the successful attempt of the Koenigsberg physiological school to invalidate the filtration theory of Leber. The physiological discussion on the destiny of the Humor aqueus leads to some concluding remarks on the idea of progress in biological sciences.

Prof. Dr. med. Gerhard Rudolph

Universität Kiel

Institut für Geschichte der Medizin und Pharmazie

Brunswiker Straße 2 a

D-2300 Kiel 